\title{
Plasma and Ion Beam Injection into an FRC
}

\author{
M. Anderson*, M. Binderbauer**, V. Bystritskii*, E. Garate*, N. Rostoker*, Y. Song*, \\ A. Van Drie*, I. Isakov*** \\ * Department of Physics and Astronomy, University of California at Irvine, CA, 92697 \\ ** Tri Alpha Energy Inc., Foothill Ranch, CA, 92610, USA \\ *** Institute of High Voltage Technology, Tomsk, Russia, 634050
}

\begin{abstract}
Experiments on the transverse injection of intense (5-20 A/cm ${ }^{2}$, wide cross section $(10 \mathrm{~cm})$, neutralized, $\sim 100 \mathrm{eV} \mathrm{H}^{+}$plasma beams, and $100 \mathrm{keV} \mathrm{H}^{+}$ion beams into a preformed B-Field Reversed Configuration (FRC) are described. The FRC background plasma had a temperature up to $\sim 5 \mathrm{eV}$ and density of $\sim 10^{13} \mathrm{~cm}^{-3}$. In contrast to earlier experiments [1], the background plasma was generated by a separate plasma gun array. Similar in general features to Pietrzuk et al. [2], the FRC startup was done using a "slow" coaxial source. Several experimental studies were conducted as a foundation to this work, including a characterization of the FRC and experiments on beam injection into a uniform B-field $[3,4]$. Results indicate that plasma beam injection into the FRC increases the B-field amplitude and duration by up to $40 \%$. The results of the ion beam injection in the FRC could be interpreted as partial beam trapping in the configuration.
\end{abstract}

\section{Introduction}

The Field Reverse Configuration (FRC) has attracted a variety of research for its potential as an alternative approach to magnetic fusion [1, 2]. Among various scenarios for the production of an FRC [1], the slow coaxial source promises to provide a long lifetime configuration. Using a slow coaxial source for FRC startup has resulted in FRC durations in the 50-100 $\mu \mathrm{s}$ range [5]. This study showed that particle and radia- tion losses impose limits on the FRC temperature and lifetime. Therefore to extend the FRC duration and increase the reversed B-field amplitude there is a need for additional continuous energy input to compensate for these losses. This paper describes the first results on the transverse injection of an intense $\mathrm{H}^{+}$plasma beam or low energy $\mathrm{H}^{+}$ion beam into a preformed FRC generated in a coaxial slow source.

\section{Experimental Setup}

The general experimental layout is shown in Fig. 1. The system consists of the main vacuum chamber with Outer (OS) and Inner (IS) coaxial solenoids, two circular plasma gun arrays located at the ends of the vessel which are used to inject background plasma along the axial B-field, plasma or ion accelerators which inject ion or plasma beams transverse to the B-field. Beam injection is done at the mid-plane of the experimental vessel and tangential to the plasma column. The OS can generate a B-field up to $2.0 \mathrm{kG}$ with a rise time of $230 \mu \mathrm{s}$. The IS can generate a B-field up to $2.5 \mathrm{~T}$ with a rise-time similar to that of the OS. By properly adjusting current amplitudes in the coils, the resulting B-field in the vessel during the first $100 \mu$ s could be as low $\sim 1 \%$ of the maximum value from the OS.

The two plasma gun arrays each consisted of 16 coaxial plasma guns with hydrated Ti electrodes saturated to a $\mathrm{H} / \mathrm{Ti}$ level of $1: 1$. They were energized from capacitor banks of up to $8 \mathrm{~kJ}$ stored energy. A total current of $\sim 120 \mathrm{kA}$ in a pulse duration of $\sim 20 \mu \mathrm{s}$

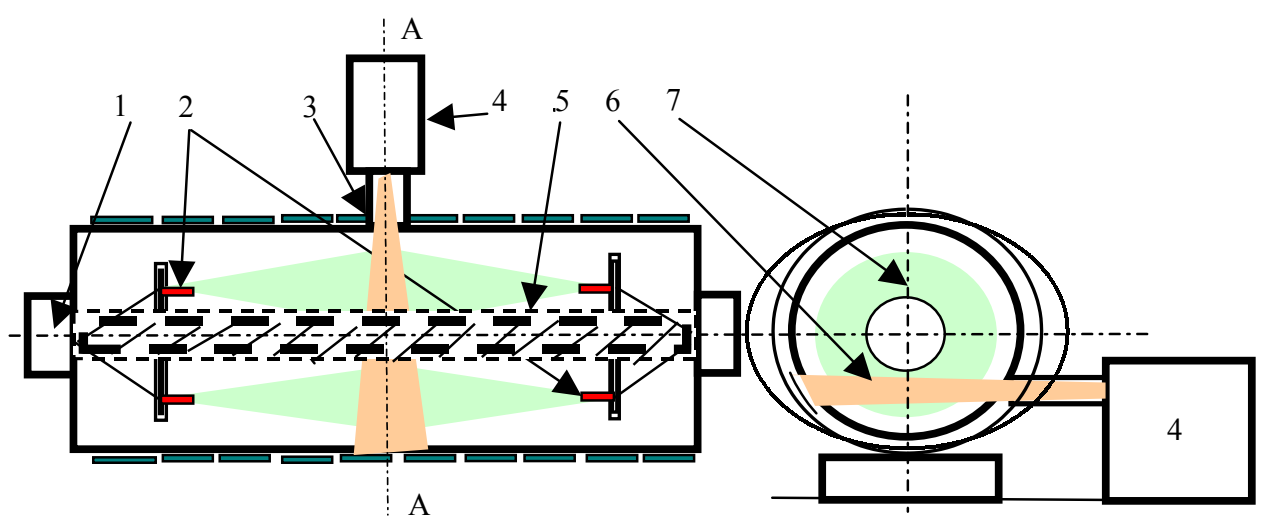

Fig. 1. Schematic of the experimental setup: 1 - plasma gun arrays power supplies, 2 - arrays of TiH plasma guns, 3 - outer solenoid, 4 - plasma/ion beam accelerator, 5 - inner solenoid, 6 - plasma/ion beam, 7 - background plasma. A median/mid-plane cross section 
energized the 32 guns. The plasma gun array provided a tubular background plasma column of $50 \mathrm{~cm}$ average diameter and $20 \mathrm{~cm}$ width. The temperature and density of the plasma at the mid-plane was a few to $10 \mathrm{eV}$ and $\sim 10^{13} \mathrm{~cm}^{-3}$ respectively. The plasma had an average streaming velocity of $(4-6) \cdot 10^{6} \mathrm{~cm} / \mathrm{s}$.

In several experimental runs the background plasma was generated by discharge in an $\mathrm{H}_{2}$ gas which was injected at the mid-plane of the vessel in a direction outward from the surface of the IS. The discharge was generated by an external one-turn coil, energized from a fast capacitor bank $\left(0.6 \mu \mathrm{F}, 17 \mathrm{kV}, t_{f}=3 \mu \mathrm{s}\right)$. The time interval between energizing the one turn coil and the OS and IS could be varied.

The plasma beam was generated and accelerated by a coaxial plasma gun, which contained a pair of coaxial "squirrel cage" conical electrodes. In some experiments the plasma gun had additional radial cable guns placed at the breach of the accelerating channel. The hydrogen flow to the breach of the gun was delivered by a fast electromagnetic puff valve. The plasma gun worked in a deflagration or snow-plow discharge mode depending on the application of the HV to the cathode. The plasma beam could be collimated and had an output diameter of 4 to $10 \mathrm{~cm}$, depending on the beam collimator size. The beam had an average divergence half angle of between 10 to $12^{\circ}$ at the entrance port to the experimental chamber. The beam plasma was injected tangentially to the background plasma column at the mid-plane of the vessel and propagated $\sim 64 \mathrm{~cm}$ in the B-field to the opposite wall. Typical Faraday cup ion signals at the mid-plane of the vessel featured a two hump structure. The ion current density in the first hump was between 10 to $20 \mathrm{~A} / \mathrm{cm}^{2}$ using the beam collimator and 30 to $60 \mathrm{~A} / \mathrm{cm}^{2}$ without collimation. This corresponds to a plasma density of 2 to $4 \cdot 10^{13} \mathrm{~cm}^{-3}$ and an ion streaming energy of $100 \mathrm{eV}$. This was followed by a second, longer trailing pulse of 20 to $30 \mu$ s duration and energy of 10 to $60 \mathrm{eV}$.

The ion beam generator produced a $2 \mathrm{kA}, 60$ to $120 \mathrm{keV}, 1 \mu \mathrm{s}$ duration $\mathrm{H}^{+}$beam of $\sim 8 \mathrm{~cm}$ diameter. The generator consisted of a $120 \mathrm{kV}, 1 \mu \mathrm{s}, 1.8 \mathrm{Ohm}$ Marx generator, a magnetically insulated ballistically focusing diode (MID), and a toroidal magnetic lens and guiding solenoid for beam transport to the entrance port of the vessel. The $\mathrm{H}^{+}$plasma source was located at the MID anode and consisted of a fast puff valve, a supersonic Laval nozzle and a shock coil, which generated an inductive discharge in the $\mathrm{H}_{2}$ gas. The distance traveled by the IB to the vessel entrance from the MID was $90 \mathrm{~cm}$. More detailed information on the design and characteristics of the IB accelerator, and ion beam parameters can be found in [3].

\section{Diagnostics}

The measurement of the B-field dynamics, ambient plasma and beam parameters were carried out using a variety of diagnostics including; Rogowski coils, axial (z) and radial (r) arrays of B-dot probes, voltage and currents monitors for the various power supplies, collimated Faraday cups, Langmuir probes, microwave cut-off probes, and $\mathrm{H}_{\alpha}$, and $\mathrm{H}_{\beta}$ time resolved spectroscopy of the background plasma.

\section{Experimental Results}

In our earlier studies of beam injection across a uniform B-field in vacuum [4] both beams propagated un-deflected with significant losses of their peripheral layers. In the case of plasma beam injection the current density in the surviving central core showed a dramatic increase. In the case of transport in the background plasma, the plasma beam did not deflect as a whole, whereas the ion beam deflection corresponded to the Larmor radius of the ion energy in the B-field. Typical waveforms for the plasma beam ion pulses, when injected in vacuum, are illustrated in Fig. 2A. Deflection of the ion beam when injected in a uniformly magnetized plasma is shown in Fig. 2 B. See [4] for a more detailed discussion on cross B field injection of plasma and ion beams in vacuum and ambient plasma.

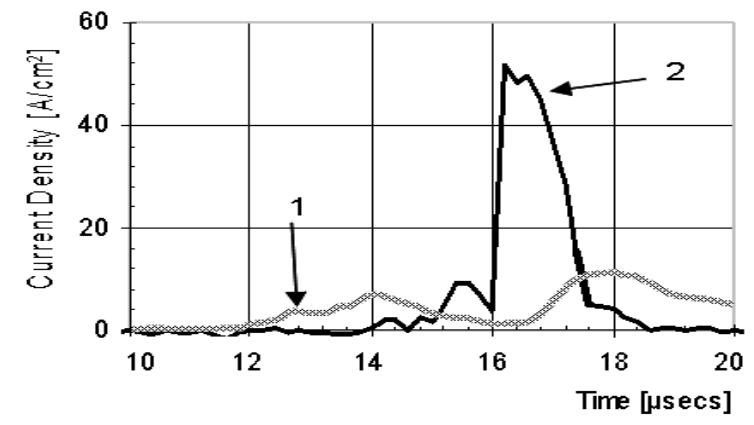

Fig. 2A. Current density waveform at the vessel mid-plane for the plasma beam propagating in vacuum (with collimator): 1 - without transverse B-field ( $100 \mathrm{eV}$ beam), 2 - with $1 \mathrm{kG}$ transverse B-field ( $\sim 30 \mathrm{eV}$ bunched beam). Zero time corresponds to triggering the current in plasma accelerator

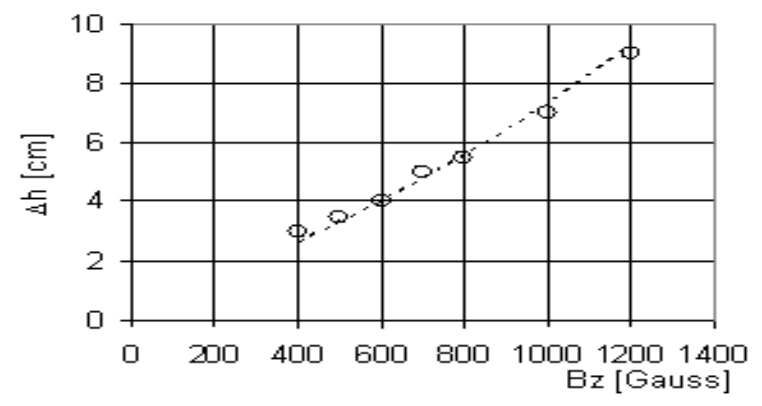

Fig. 2B. Vertical deflection $(\Delta \mathrm{h})$ of the IB in the mid-plane of the vessel from the line of injection after propagating in ambient plasma across the B-field as a function of B-field amplitude. The circles represent experimental data and the broken line corresponds to one particle calculations for a $60 \mathrm{keV}$ beam. 


\subsection{FRC formation}

The FRC was formed by firing the background plasma gun arrays 10 to $20 \mu \mathrm{s}$ prior to energizing the OS and IS. Due to the changing flux from the solenoids, the azimuthal current induced in the plasma column provided decoupling of the OS and IS B-fields in the vessel, which resulted in the FRC formation. In a typical pulse the FRC appeared between 10 to $20 \mu$ s after energizing the OS and IS and reached the maximum FRC field in 30 to $60 \mu \mathrm{s}$. The FRC lasted as long as the induced azimuthal E-field, resulting from $\mathrm{d} I / \mathrm{d} t$ in the IS, could support the azimuthal current in the background plasma.

Typical waveforms from the $\mathrm{B}_{\mathrm{z}}$-dot arrays, as well as a current pulse from one of the plasma guns used to generate the background plasma and currents in the OS and IS are illustrated in Fig. 3.

\subsection{PB injection in the FRC}

Table I illustrates the main changes in the FRC characteristics when injecting the plasma beam. In the table the resulting amplitude of the B-field in the FRC, and its duration, are given for several time delays between the plasma beam injection relative to the background plasma injection. The data corresponds to the combination of currents in the OS and IS which generally resulted in the best FRC characteristics. As can be seen from the table, plasma beam injection pro- vided a longer duration FRC with a higher B-field amplitude. The maximum increase of the FRC reversed B-field amplitude, due to plasma beam injection, was $50 \%$.

\subsection{IB injection in the FRC}

It is worth noting that the injected ion beam particle current and density $\left(\sim 2 \mathrm{kA}, \sim 10^{11} \mathrm{~cm}^{-3}\right)$ were 10 times and 1000 times lower, respectively, than the current and density values in the FRC plasma ( $\sim 20$ to $30 \mathrm{kA}, \sim 10^{14} \mathrm{~cm}^{-3}$ ). These factors made it impossible to directly measure the captured IB fraction in the FRC because the expected changes in the probe signals were in the range of the shot-to-shot statistical variations. However we could compare the data on ion beam propagation in the FRC plasma with our previous results on ion beam propagation in a magnetized plasma of density $>10^{13} \mathrm{~cm}^{-3}$ at similar B-field values. In those experiments, at $\mathrm{B}=0.6 \mathrm{kG}$, collimated Faraday cup (CFC) arrays measured current densities of $\sim 5 \mathrm{~A} / \mathrm{cm}^{2}$. The Faraday cups were placed along the Larmor $\mathrm{jxB}$ trajectory corresponding to the ion beam energy of 60 to $90 \mathrm{keV}$. However, when the IB was injected into the preformed FRC with a similar B-field amplitude, the measured IB density was a factor of about 10 times smaller. This result could be interpreted as partial trapping of the ion beam in the FRC configuration. More detailed studies are planned.

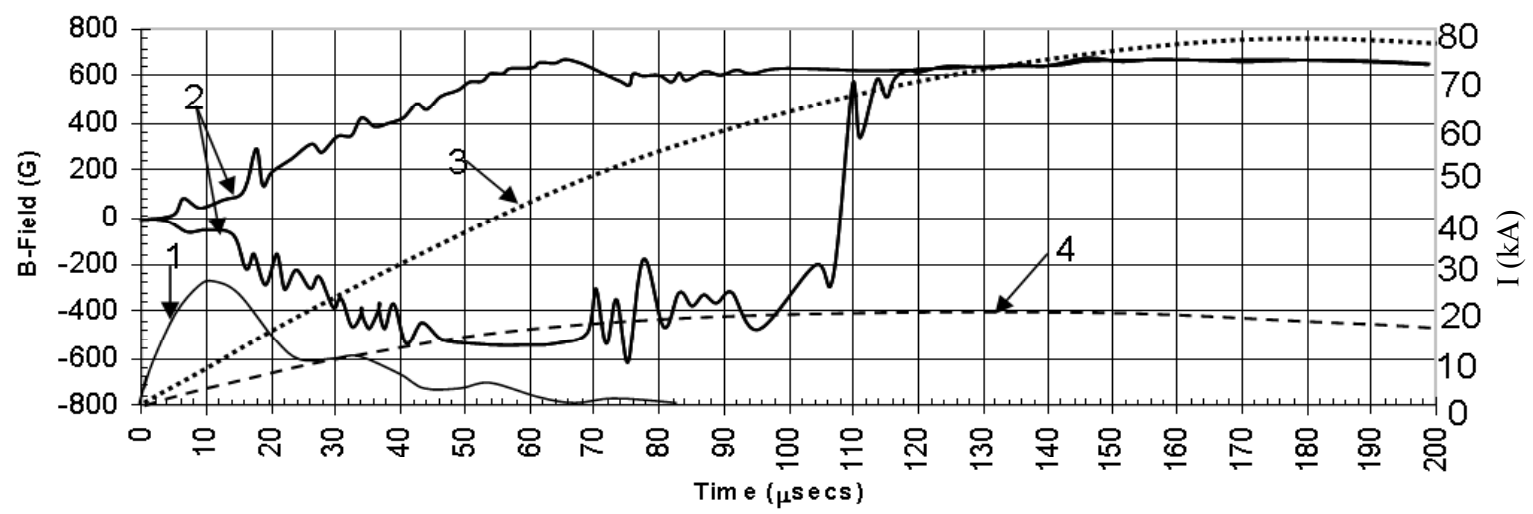

Fig. 3. Typical waveforms during FRC formation from respective diagnostics as a function of time: $1-\mathrm{PG}$ array current pulse; $2-\mathrm{B}_{\mathrm{z}}$-field in the median cross section of the vessel at the radii $12 \mathrm{~cm}$ - upper trace, and $39 \mathrm{~cm}$ - lower trace (B-dot array data); 3 - current in the internal solenoid; 4 - current in the external solenoid

Table I. FRC characteristics for different time delays between background plasma generation and plasma beam injection

\begin{tabular}{|c|c|c|c|c|}
\hline Conditions & $\begin{array}{c}\text { BGP } \\
\text { only }\end{array}$ & $\begin{array}{c}\text { BPG \& PA } \\
\delta t=90 \mu \mathrm{s}\end{array}$ & $\begin{array}{c}\text { BPG \& PA } \\
\delta t=110 \mu \mathrm{s}\end{array}$ & $\begin{array}{c}\text { BPG \& PA } \\
\delta t=130 \mu \mathrm{s}\end{array}$ \\
\hline $\begin{array}{c}\text { FRC Duration }[\mu \mathrm{s}] \\
\text { Average }\end{array}$ & 130 & 148 & 145 & 150 \\
\hline $\begin{array}{c}\mathrm{B}_{\mathrm{z}} \text {-field [Gauss] } \\
\text { Average }\end{array}$ & 200 & 300 & 288 & 260 \\
\hline $\begin{array}{c}\mathrm{B}_{\mathrm{z}}[\mathrm{R}] \text { field [Gauss] } \\
\text { Average }\end{array}$ & 120 & 188 & 150 & 138 \\
\hline
\end{tabular}




\section{Conclusion}

Injection of intense Plasma beams in an FRC resulted in an increase of the reversed B-field amplitude and extension of the FRC lifetime. This could be due to additional heating of the plasma resulting in an increase of plasma conductivity.

The results of our measurements on the injection and propagation of an ion beam into an FRC could be interpreted as partial beam capture in the FRC. More detailed studies of the particle losses and other causes of FRC lifetime limitations are currently under way.

\section{Acknowledgements}

The authors would like to acknowledge fruitful discussions with, and computational help from, Drs. S. Dettrick and A. Querushi. The authors also gratefully acknowledge the technical support of
S. Armstrong, M. Morehouse, K. Walters and G. Strashnoy. This work was carried out at the University of California Irvine under contract and in cooperation with Tri Alpha Energy, Inc.

\section{References}

[1] Tuszewsky, Nuclear Fusion 28, 2033 (1988).

[2] Z.A. Pietrzyk, G. Vlases, R. Brooks , K.D. Hahn, R. Raman, Nuclear Fusion 27, 1478 (1987).

[3] M. Anderson, V. Bystritskii, E. Garate et al., IEEE Pulsed Power, October 2004 (in print); Beams, Saint-Petersburg, July 2004 (in print).

[4] M. Anderson, V. Bystritskii, E. Garate et. al., Proc. Intern. Top. Conf. of High Power Particle Beams, Saint-Petersburg, July 2004 (in print).

[5] J.T. Slough, K.E. Miller, Phys. Rev. Lett. 85, 144 (2000). 